\title{
Complexity Analysis of Optimal Recharge Scheduling for Electric Vehicles
}

\author{
Cristina Rottondi, Member, IEEE, Giovanni Neglia, Member, IEEE, and Giacomo Verticale Member, IEEE
}

\begin{abstract}
The massive introduction of Electric Vehicles (EVs) will make fleet managers spend a significant amount of money to buy electric energy. If energy price changes over time, accurate scheduling of recharging times may result in significant savings. In this paper we evaluate the complexity of the optimal scheduling problem considering a scenario with a fleet manager having full knowledge of the customers' traveling needs at the beginning of the scheduling horizon. We prove that the problem has polynomial complexity and provide complexity lower and upper bounds. Moreover, we propose an online sub-optimal scheduling heuristic that schedules the EVs' recharge based on historical travelling data. We compare the performance of the optimal and sub-optimal methods to a benchmark online approach that does not rely on any prior knowledge of the customers' requests, in order to evaluate whether the additional complexity required by the proposed strategies is worth the achieved economic advantages. Numerical results show up to of $35 \%$ cost savings with respect to the benchmark approach.
\end{abstract}

Index Terms-Electric Vehicles; Vehicle-to-Grid Interactions; Optimal Recharge Scheduling;

\section{INTRODUCTION}

Battery/fuel cell-powered vehicles and hybrid automobiles are expected to play a pivotal role in the novel Smart Grid scenario, concurring in reducing carbon emissions by fostering the exploitation of Renewable Energy Sources (RESes). The potential consequences of a widespread introduction of Electric Vehicles (EVs) have been recently investigated [1]: on one hand the EVs plugged at charging stations significantly increase the power load experienced by the grid, on the other hand the storage capacities of their batteries could also be exploited to cope with the intermittent energy generation patterns of RESes by recharging them in presence of surpluses in energy production [2]. An entity which is responsible for intelligently scheduling the charging/discharging process of a large number of EVs [3] can coordinate such Vehicle-to-Grid (V2G) interactions according to various business models [4], possibly considering economic incentives to compensate the additional battery deterioration due to frequent charge/discharge or to encourage the owners to plug their EV when parked. The reader is referred to [5] for a detailed overview on the technoeconomical models of load aggregation agents for EVs.

Therefore, V2G has recently gained increasing interest in various disciplines related to Information and Communication Technologies (ICT), ranging from computer science to

A preliminary version of this paper has been published as C. Rottondi, G. Neglia, G. Verticale. "On the Complexity of Optimal Electric Vehicles Recharge Scheduling.” In Proc. IEEE Online GreenComm 2014.

This work has been partially funded by Regione Lombardia under grant no. 40545387 Smart Campus as Urban Open LAbs(SCUOLA). telecommunications and control theory: numerous optimal and heuristic scheduling strategies have been investigated, mostly aimed at reducing carbon emissions [6], [7] or the overall recharge cost in presence of time-variable energy tariffs (see e.g. [8]-[10]). Privacy-preserving solutions to perform the battery recharge scheduling without disclosing the travelling habits and battery level details of the users have also been proposed [11]. The control-oriented research community thoroughly investigated the development of on-board controllers for the recharging process [6], [12] and the stochastic modeling of the individual EV behavior [13]. Conversely the telecommunications community focused primarily on the characterization of the overall system in terms of interactions among the various stakeholders (EVs, load aggregator, energy utility), assuming either a centralized scheduler [14] or a set of aggregators operating in a distributed scenario [9].

A substantial body of work investigates optimal and heuristic policies for the battery recharge of a population of EVs. Among the most remarkable contributions, Han et al. [8] formulate a game model for $\mathrm{V} 2 \mathrm{G}$ interactions in presence of a profit-driven recharging station and two coexisting sets of EVs, behaving respectively as selfish or cooperative: the recharge of the former set of EVs is decided by the customers themselves according to the real-time energy selling price, while the station can directly control the charging/discharging process of the EVs belonging to the latter set. A game theoretical framework is adopted also by Zou et al. [15], who design a distributed charging coordination method for EVs relying on an auction mechanism based on progressive second price. A distributed approach aimed at increasing scalability is applied by Rivera et al. [16]: they propose a decentralized optimization algorithm based on the Alternating Direction Method of Multipliers and separate the centralized optimal fleet charging problem into individual optimization problems for the single EVs, which are coupled and solved consistently by exchanging incentive signals between them, plus one aggregated problem that optimizes fleet goals. The framework can be parameterized to trade-off the importance of fleet goals versus the objectives of the individual EVs. Conversely, since our scenario assumes that the entire fleet is owned by a unique entity, competitiveness among the vehicles is not considered and thus the objective function models only the aggregator's utility.

He et al. [9] propose a convex optimization model for a global scheduling optimization problem aimed at the minimization of the recharging cost assuming full knowledge of 
the future behavior of the customers. The authors compare it to a distributed version that takes into account dynamic arrivals and finds local optima for subsets of vehicles. They show that this version achieves close-to-optimum performance. However, the authors assume that the periods in which each vehicle is plugged and available for recharging are problem inputs, while our model optimizes the assignment between vehicles and users to be served, with the aim of minimizing the recharge costs. The approach adopted in our work also compares the global optimum achievable by means of an Integer Linear Program (ILP) formulation run by an omniscient scheduler to the results obtained by a sub-optimal online scheduling.

Joe-Wong et al. [17] combine a convex optimization formulation for computing day-ahead energy prices and an algorithm for estimating and refining EVs' user reaction to the prices. The algorithm allows the provider to dynamically adjust the offered prices based on the EVs' behavior. Conversely, our optimization scenario does not allow for price negotiation, since it is based on day-ahead tariffs.

Chenrui et al. [18] address the EV recharge scheduling problems by considering both a static and a dynamic scenario: in the former, customers' charging demands are provided to the aggregator in advance, whereas in the latter vehicle arrivals and departures are not known to the aggregator in advance. The authors present linear programming (LP)-based optimal schemes for the static problems and heuristic algorithms for the dynamic problems. In our paper, we adopt the same approach. However, that work does not address the complexity evaluation of the proposed scheduling techniques, which is the aim of our contribution.

Li et al. [10] discuss a methodology for modeling the overall charging demand of EVs based on queuing theory, which allows for the differentiation of the fleet behavior in case of a charging station and a local residential community. A queuing theory-based approach is used also by Alizadeh et al. [14] and Zhang et al. [19] to provide a stochastic mathematical model for EV aggregate load aimed at shortterm load forecasting. Paper [10] assumes a maximum limit on the number of vehicles contemporaneously charged, whereas paper [14] assumes an infinite number of available plugs. In our work, we adopt the latter assumption, which does not introduce additional waiting times in the recharging process.

This paper investigates the cost savings achievable by an optimal centralized scheduling strategy minimizing the overall recharging cost. The considered scenario is a day-ahead planning, that assumes knowledge of the details about the customers' travelling needs and the day-ahead energy price. The contribution of the paper is twofold: first, we propose a planning algorithm which is used by a service provider (e.g. car rental, logistics/transportation company owning a fleet of EVs) to obtain the EV-to-user assignment and the battery recharge scheduling that minimizes the overall recharge cost in presence of variable energy prices. To the best of our knowledge, this is the first work which jointly optimizes the assignment of the EVs to users' trips and the schedule of the EV charging periods. We prove that the problem has polynomial complexity and provide complexity lower and upper bounds.
The drawback of such optimal scheduling approach is that it requires prior knowledge of the travelling patterns of the users, which could be impractical to be obtained in real scenarios. As a comparison, we propose an online scheduling heuristic, which relies exclusively on statistics of the departure process obtained from historical data. To assess the performance of our proposed optimal and sub-optimal approaches, we evaluate the achieved cost savings w.r.t. an online benchmark scheduling strategy that does not rely on prior knowledge of the users' requests nor aims to minimize the total recharge costs, but simply finds a feasible recharge plan fulfilling the users' travelling needs, with a computational complexity exhibiting linear dependency on the problem input size.

The remainder of the paper is structured as follows: Section II recalls some background notions, whereas Section III defines the scheduling problem, proves that it can be solved to the optimum in a number of operations polynomially depending on the problem input size, describes an optimal algorithm for its solution, and provides complexity upper and lower bounds. The online heuristic and the benchmark approach are presented in Section IV. Section V evaluates the cost savings achieved by the optimal solution approach w.r.t. the online heuristic approach and the benchmark technique. Finally, we draw our conclusions in the last Section.

\section{BACKGROUND}

In this Section we shortly describe the Minimum Weighted Matching Problem (MWMP) in a complete balanced bipartite graph, which constitutes one of the building blocks of our proposed scheduling mechanisms. Let $G=\left(N_{1}, N_{2}\right)$ be a graph whose vertexes can be divided into two disjoint subsets $N_{1}, N_{2}$ such that $\left|N_{1}\right|=\left|N_{2}\right|$ and that each vertex $n_{1} \in N_{1}$ is connected to every vertex $n_{2} \in N_{2}$ by an edge $\left(n_{1}, n_{2}\right) \in E$ with non-negative weight $w\left(n_{1}, n_{2}\right)$. The problem goal is to individuate a perfect matching $\bar{E}$ of minimum weight for graph $G$, i.e. a set of $\left|N_{1}\right|$ edges connecting each node $n_{1} \in N_{1}$ to a different node $n_{2} \in N_{2}$ such that from each vertex exactly one edge starts and the sum of the weights associated to these edges is minimum.

\section{Formulation:}

$$
\min _{\bar{E}} \sum_{\left(n_{1}, n_{2}\right) \in \bar{E}} w\left(n_{1}, n_{2}\right)
$$

Subject to:

$$
\begin{gathered}
\forall n_{1} \in N_{1} \exists n_{2} \in N_{2}:\left(n_{1}, n_{2}\right) \in \bar{E} \\
\forall n_{2} \in N_{2} \exists n_{1} \in N_{1}:\left(n_{1}, n_{2}\right) \in \bar{E} \\
|\bar{E}|=\left|N_{1}\right|=\left|N_{2}\right|
\end{gathered}
$$

The objective function minimizes the total weight of the selected edges. Constraints (1) and (2) impose that each vertex in $N_{1}$ is connected to a vertex in $N_{2}$ by one of the edges in $\bar{E}$ (and vice versa), whereas Constraint (3) sets the size of $\bar{E}$ to the cardinality of $N_{1}$ and $N_{2}$, which ensures that the selected edges form a matching. Therefore, the above formulation individuates the perfect matching of minimum weight for graph $G$. 
The MWMP problem has been proved to be solved to the optimum in $O\left(\left|N_{1}\right|^{3}\right)$ operations by means of the so called Hungarian algorithm [20]. More efficient algorithms with complexity between $O\left(\left|N_{1}\right|^{2}\right)$ and $O\left(\left|N_{1}\right|^{3}\right)$ can be applied by making additional assumptions on the maximum weight value or by relaxing the assumption of completeness of the graph [21].

\section{Formulation And Solution of the Scheduling PROBLEM (SP)}

\section{A. Problem Formulation}

We consider a scenario in which a service provider owns a fleet of vehicles $\mathcal{V}$ and a recharging station where the vehicles are parked and can be recharged after usage. We assume that the station is equipped with a sufficient number of plugs to recharge the vehicles at any time without queuing. Each vehicle is characterized by the same battery charging rate $r$. Note that the recharge operations are assumed to be non-interruptible, meaning that, once the recharge process has started, it must be completed without intermediate breaks. We also assume that time is divided in epochs of duration $\Delta$. Let, $\mathcal{U}$ and $\mathcal{T}$ be the set of users and the set of discretized epochs within the optimization time span, respectively. Before the beginning of the scheduling horizon, every user $u \in \mathcal{U}$ specifies a service request in terms of the triplet $\left(t_{u}^{d}, t_{u}^{a}, e_{u}\right)$, indicating the user's departure and arrival time $\left(0<t_{u}^{d}<t_{u}^{a}<|\mathcal{T}|\right)$ and the amount of energy discharged from the battery during the travel, respectively. ${ }^{1}$ Without loss of generality, we assume that each user expresses a single service request during the optimization period. Moreover, we assume that at epoch $t=0$ all the vehicles are parked at the recharging station with fully charged batteries. A vehicle returning to the charging station after serving a customer must be fully recharged before being assigned to a new user. At the end of the scheduling period $(t=|\mathcal{T}|)$, every vehicle must be back to the recharging station and the recharge of its battery must be completed, in order to be ready to serve another user by the beginning of the next scheduling horizon.

Let $c_{\tau} \geq 0$ be the energy price for each epoch $\tau \in \mathcal{T}$. A possible schedule for the recharge of the set of vehicles $\mathcal{V}$ to serve the set of users $\mathcal{U}$ during the set of time epochs $\mathcal{T}$ is defined by the set $\overline{\mathcal{Z}} \subseteq \mathcal{Z}=\mathcal{U} \times \mathcal{V} \times \mathcal{T}$ of $|\mathcal{U}|$ triplets $(u, v, t)$, indicating the starting epoch $t$ for the recharge of vehicle $v$ serving user $u$. The goal of the problem is to individuate the feasible scheduling ensuring the lowest overall recharge cost experienced by the service provider. Note that the pattern of time-variable energy cost $c_{t}$ can be designed according to different criteria, e.g. the maintenance of the balancing between energy generation and usage within the power grid. In such case, increasing the energy price during periods of peak energy consumption or of lack of energy production from renewable sources automatically discourages the recharge of the vehicles (unless strictly required to serve incoming users).

\footnotetext{
${ }^{1}$ The battery discharge associated to the travel can be computed as a function of the expected travelling distance. Note that the proposed scheduling approach is agnostic w.r.t. the technique adopted for the computation of such amount.
}

Conversely, the energy tariff can be lowered in case of excess of energy production (or lack of power load) experienced by the grid, in order to incentivize energy absorption by charging the vehicles currently parked at the recharging stations.

\section{Formulation:}

$$
\min _{\overline{\mathcal{Z}} \subseteq \mathcal{Z}} \sum_{(u, v, t) \in \overline{\mathcal{Z}}} \sum_{k=t}^{t+\left\lceil\frac{e_{u}}{r}\right\rceil-1} c_{k}
$$

subject to:

$$
\left|\mathcal{Z}_{u}\right|=1 \quad \forall u \in \mathcal{U}
$$

where $\mathcal{Z}_{u}=\left\{\left(u^{\prime}, v, t\right) \in \overline{\mathcal{Z}}: u^{\prime}=u\right\}$.

$$
\begin{gathered}
t \geq t_{u}^{a} \quad \forall(u, v, t) \in \overline{\mathcal{Z}} \\
t \leq t_{u^{\prime}}^{d}-\left\lceil\frac{e_{u}}{r}\right\rceil \forall\left((u, v, t),\left(u^{\prime}, v, t^{\prime}\right)\right) \in \overline{\mathcal{Z}} \times \overline{\mathcal{Z}}: t_{u}^{a} \leq t_{u^{\prime}}^{d}
\end{gathered}
$$

The objective function minimizes the overall recharging cost experienced by the service provider. Constraint (5) ensures that the set of vehicles serving a given user has cardinality 1, i.e. that each user is associated to exactly one vehicle. Constraint (6) imposes that a vehicle is charged only after its return to the charging station and Constraint (7) states that vehicle $v$ associated to a given user $u$ can serve a second user $u^{\prime}$ only in case $u^{\prime}$ departs after the return of $u$ and there is sufficient time to complete the battery recharge before the departure of $u^{\prime}$.

In the following Subsections we prove the computational equivalence of SP and MWMP by showing that SP is polynomially reducible to MWMP and vice versa. Moreover, we provide upper and lower bounds for the complexity of SP.

\section{B. Resolution Procedure of the Scheduling Problem}

In order to find the optimal solution of a generic instance of the SP problem, we first transform it into a corresponding instance of the MWMP problem. Let $N_{1}^{\mathcal{U}}, N_{2}^{\mathcal{U}}$ be two disjoint sets of nodes of cardinality $|\mathcal{U}|$ each, representing the users' arrivals and departures, respectively. Moreover, let $N_{1}^{\mathcal{V}}, N_{2}^{\mathcal{V}}$ be two disjoint sets of nodes of cardinality $|\mathcal{V}|$, representing the fleet of EVs parked at the charging station at times $t=0$ and $t=|\mathcal{T}|$, respectively. Finally, we define the sets $N_{1}=N_{1}^{\mathcal{U}} \cup N_{1}^{\mathcal{V}}, N_{2}=N_{2}^{\mathcal{U}} \cup N_{2}^{\mathcal{V}}$ and consider the complete balanced bipartite graph $G=\left(N_{1}, N_{2}\right)$. Links have non-negative weights defined as follows.

$$
\text { If } n_{1} \in N_{1}^{\mathcal{V}} \text {, then } w\left(n_{1}, n_{2}\right)=0 \text {. }
$$$$
\text { If } n_{1} \in N_{1}^{\mathcal{U}} \text { and } n_{2} \in N_{2}^{\mathcal{V}} \text {, then }
$$

$$
w\left(n_{1}, n_{2}\right)=\min _{t_{n}^{a} \leq t \leq|\mathcal{T}|-\left\lceil\frac{e_{n_{1}}}{r}\right\rceil} \sum_{k=t}^{t+\left\lceil\frac{e_{n_{1}}}{r}\right\rceil-1} c_{k} .
$$

If $n_{1} \in N_{1}^{\mathcal{U}}, n_{2} \in N_{2}^{\mathcal{U}}$, and $t_{n_{1}}^{a} \leq t_{n_{2}}^{d}-\left\lceil\frac{e_{n_{1}}}{r}\right\rceil$, then

$$
w\left(n_{1}, n_{2}\right)=\min _{t_{n_{1}}^{a} \leq t \leq t_{n_{2}}^{d}-\left\lceil\frac{e_{n_{1}}}{r}\right\rceil} \sum_{k=t}^{t+\left\lceil\frac{e_{n_{1}}}{r}\right\rceil-1} c_{k} .
$$

Finally, $w\left(n_{1}, n_{2}\right)=\infty$ in all other cases. A pictorial view of $G$ is proposed in Figure 1 . 


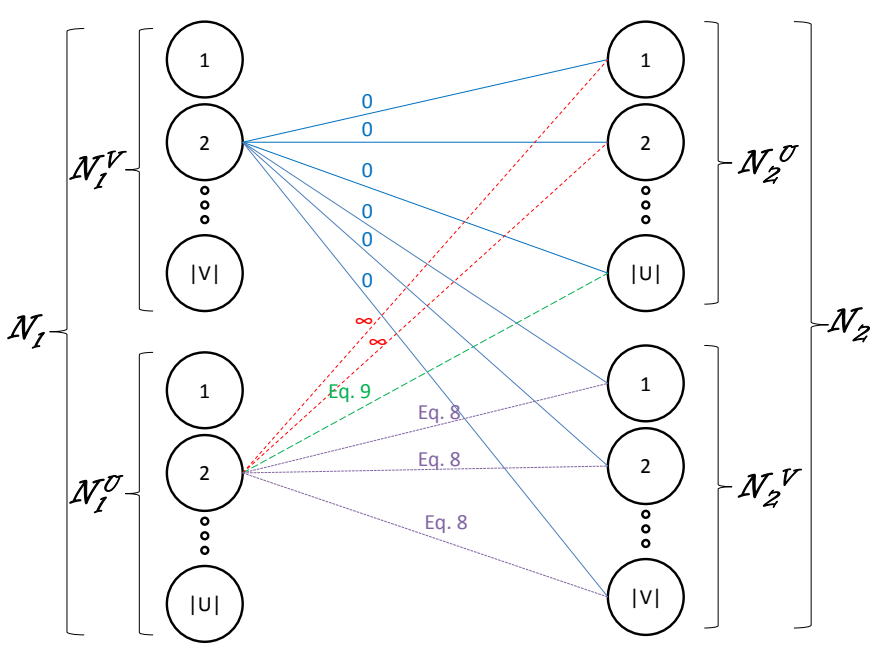

Fig. 1. Complete balanced bipartite graph obtained from the SP problem. Weights of the outgoing edges from the nodes corresponding to vehicle $v=2$ and user $u=2$ are shown as example.

Each link corresponds to a possible scheduling choice and its weight is the associated battery recharge cost. An edge connecting a node $n_{1} \in N_{1}^{\mathcal{V}}$ to a node $n_{2} \in N_{2}^{\mathcal{V}}$ corresponds to a vehicle that is not used during the scheduling period, thus its weight is 0 . An edge between a node $n_{1} \in N_{1}^{\mathcal{V}}$ and a node $n_{2} \in N_{2}^{\mathcal{U}}$ denotes that $n_{2}$ is the first user of vehicle $n_{1}$. The vehicle does not need to be charged (because all vehicles are fully charged at the beginning), therefore, also in this case, the associated weight is 0 . Conversely, an edge $\left(n_{1}, n_{2}\right)$ with $n_{1} \in N_{1}^{\mathcal{U}}, n_{2} \in N_{2}^{\mathcal{V}}$ corresponds to user $n_{1}$ being the last user for vehicle $n_{2}$, so its weight is the minimum energy cost associated to a recharge period of $\left\lceil\frac{e_{n}}{r}\right\rceil$ slots, chosen during the interval $\left[t_{n_{1}}^{a},|\mathcal{T}|\right]$, i.e. the time window between the return of the customer $n_{1}$ and the end of the scheduling horizon (the vehicle $n_{2}$ must be charged after serving its last customer, in order to be fully charged before the beginning of the next scheduling period). This cost is expressed by (8). Finally, an edge between $n_{1} \in N_{1}^{\mathcal{U}}$ and $n_{2} \in N_{2}^{\mathcal{U}}$ denotes that the same vehicle is consecutively used for users $n_{1}$ and $n_{2}$. This is not possible if the time interval between the return of user $n$ and the departure of user $n_{2}$ is not long enough to guarantee that the vehicle can be fully recharged (i.e. if $t_{n_{1}}^{a}>t_{n_{2}}^{d}-$ $\left.\left\lceil e_{n_{1}} / r\right\rceil\right)$. In such case the weight of edge $\left(n_{1}, n_{2}\right)$ is set to $\infty$. Otherwise, if the interval is long enough, Equation (9) sets the cost of the edges $\left(n_{1}, n_{2}\right)$ to the minimum energy cost associated to a recharge period of $\left\lceil\frac{e_{n_{1}}}{r}\right\rceil$ slots chosen in the period $\left[t_{n_{1}}^{a}, t_{n_{2}}^{d}-1\right]$ (which is the time span between the return of user $n_{1}$ and the departure of user $n_{2}$ ). The asymptotic complexity of computing the weights is $O\left(|\mathcal{T}|(|\mathcal{U}|+|\mathcal{V}|)^{2}\right)$, which shows a polynomial dependency on $|\mathcal{T}|,|\mathcal{U}|$, and $|\mathcal{V}|$.

Once the mapping is completed, the minimum weight matching over graph $G$ can be computed by means of the Hungarian algorithm (or any other state-of-the-art approach). The algorithm output $\bar{E}$ can be converted in a solution $\bar{Z}$ of the SP instance by means of Algorithm 1, which runs in $O(|\mathcal{U}||\mathcal{T}|)$ operations. The algorithm considers the vehicles one at a time (lines 2-11), sequentially visits in chronological order

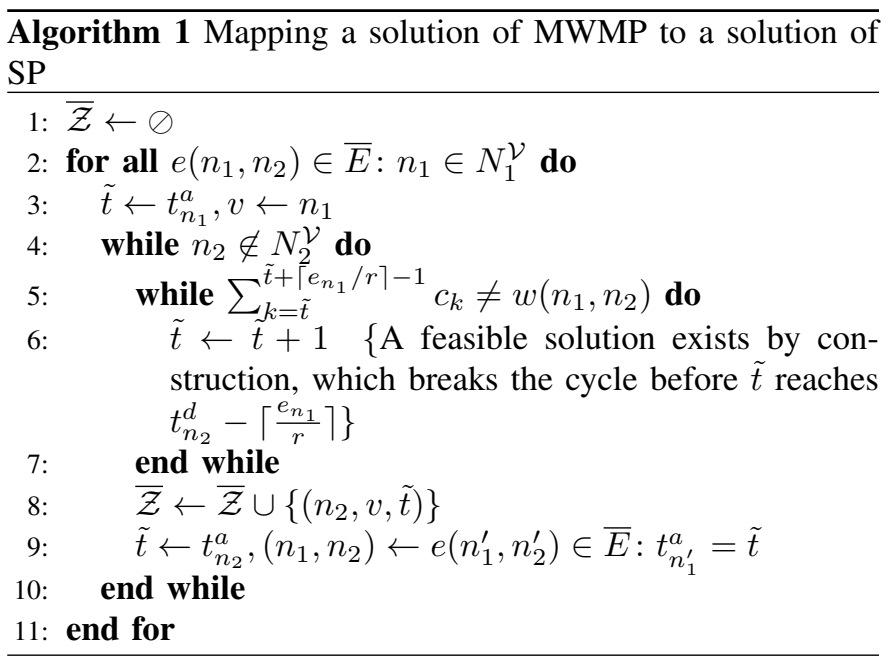

the edges of the matching $\bar{E}$ which correspond to the recharge periods scheduled for vehicle $v$ (lines 4-10), and adds to the set $\overline{\mathcal{Z}}$ the associated triplets (line 8). Note that, if the matching $\bar{E}$ has finite weight, by construction the corresponding scheduling $\overline{\mathcal{Z}}$ has the same cost. If the matching has infinite cost, then it contains at least one edge with infinite weight and we can conclude that the corresponding schedule is not feasible.

Theorem 1: Given a generic instance of the SP problem and the corresponding MWMP problem over the complete balanced bipartite graph $G\left(N_{1}, N_{2}\right)$, a scheduling $\overline{\mathcal{Z}}$ is optimal if and only if its corresponding matching has minimum weight.

Proof: We start observing that for any arbitrary schedule $\overline{\mathcal{Z}}$ there exists a matching $\bar{E}$ with cost no higher than $\overline{\mathcal{Z}}$, which can be obtained starting from $\overline{\mathcal{Z}}$ and applying Algorithm 2: for each vehicle $v \in \mathcal{V}$, the algorithm identifies the set of users who drive it (line 3), sorts them in ascending order w.r.t. their respective recharge starting times (line 5), and includes in the set $\bar{E}$ the edges with weight equal to the corresponding recharge cost (line 6-15). Since $\overline{\mathcal{Z}}$ is a feasible solution of the SP problem, by construction it holds that $n_{1} \neq n_{1}^{\prime}, n_{2} \neq n_{2}^{\prime}$ for all $\left(n_{1}, n_{2}\right),\left(n_{1}^{\prime}, n_{2}^{\prime}\right) \in \bar{E}$, and that for each $n_{1} \in N_{1}\left(n_{2} \in\right.$ $\left.N_{2}\right)$ there exists a node $n_{2} \in N_{2}\left(n_{1} \in N_{1}\right)$ such that the edge $\left(n_{1}, n_{2}\right)$ belongs to set $\bar{E}$. It follows the set of $\left|N_{1}\right|$ sequences identifies a matching $\bar{E}$ on $G$, according to the interpretation of the different kind of links we have provided in Section III-B (e.g. if users $n_{1}$ and $n_{2}$ consecutively drive vehicle $v$, then the edge between $n_{1} \in N_{1}^{\mathcal{U}}$ and $n_{2} \in N_{2}^{\mathcal{U}}$ is included in the matching $\bar{E}$ ). We observe that different schedules can produce the same set of ordered sequences of users (these schedules only differ for the time epochs when the recharge periods start). The weights of the links in $\bar{E}$ correspond to the minimum recharge costs that can be obtained under the constraint that each vehicle is assigned that specific sequence of users. It follows that the matching $\bar{E}$ has cost no higher than the scheduling $\overline{\mathcal{Z}}$.

Let $\overline{\mathcal{Z}}_{\text {opt }}$ be the optimal solution of a given instance of SP, with cost

$$
\Xi=\sum_{(u, v, t) \in \overline{\mathcal{Z}}_{o p t}} \sum_{k=t}^{t+\left\lceil e_{u} / r\right\rceil-1} c_{k} .
$$




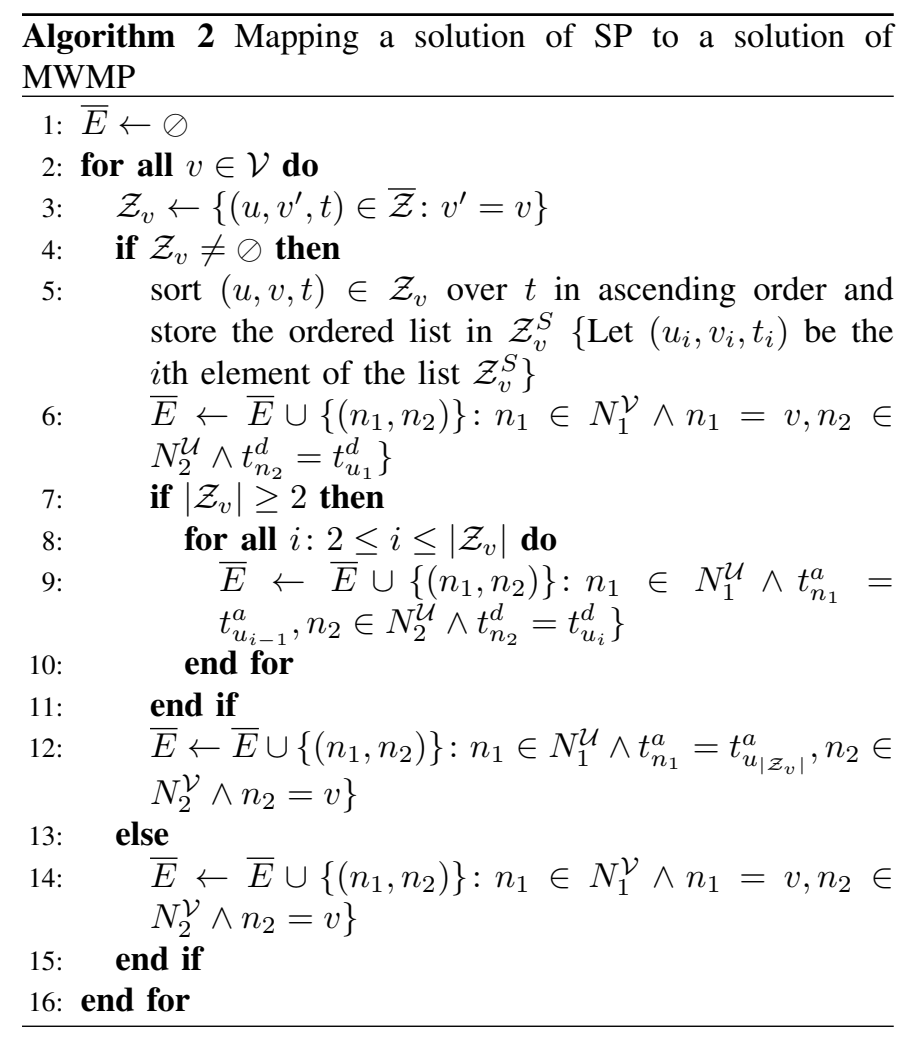

Let $\bar{E}_{\text {opt }}$ be the solution of the corresponding MWMP instance obtained by means of Algorithm 2, having cost no higher than $\Xi$. If $\bar{E}_{\text {opt }}$ is not the optimal solution of MWMP, then there exists a solution $\bar{E}_{\text {opt }}^{*}$ with cost $\Xi^{*}<\Xi$. On input of $\bar{E}_{\text {opt }}^{*}$, Algorithm 1 computes a scheduling $\overline{\mathcal{Z}}_{\text {opt }}^{*}$ of cost $\Xi^{*}$, which contradicts the hypothesis of optimality of $\overline{\mathcal{Z}}_{\text {opt }}$.

Analogously, let $\bar{E}_{\text {opt }}$ be the matching of minimum weight of an instance of MWMP constructed as in Section III-B, with $\operatorname{cost} \Gamma=\sum_{\left(n_{1}, n_{2}\right) \in \bar{E}_{\text {opt }}} w\left(n_{1}, n_{2}\right)$. Let $\bar{Z}_{\text {opt }}$ be the solution of the original SP instance obtained by Algorithm 1 on input of $\bar{E}_{\text {opt }}$, which has cost no higher than $\Gamma$. If there exists a scheduling $\overline{\mathcal{Z}}_{\text {opt }}^{*}$ with cost $\Gamma^{*}<\Gamma$, then Algorithm 2 computes a matching $\bar{E}_{\text {opt }}^{*}$ of cost $\Gamma^{*}$, which implies that $\bar{E}_{\text {opt }}$ is not optimal.

It follows that for a given instance of SP, a scheduling $\overline{\mathcal{Z}}$ obtained by mapping a matching $\bar{E}$ computed over the corresponding instance of MWMP by means of Algorithm 1 is optimal iff $\bar{E}$ is the matching of minimum weight.

\section{Complexity Evaluation of $S P$}

We now derive upper and lower bounds for the complexity of the SP problem. To do so, we first prove the computational equivalence of SP and MWMP by means of the following three theorems.

Theorem 2: The SP and MWMP problems are computationally equivalent, i.e. $\mathrm{SP} \equiv{ }_{P} \mathrm{MWMP}$

Proof: The proof that the SP problem is polynomially reducible to the MWMP problem, i.e. SP $\leq_{P} \mathrm{MWMP}$, consists in a Cook reduction which can be straightforwardly derived from the proof of Theorem 1. Moreover, the proof that the MWMP problem is polynomially reducible to the
SP problem, i.e. MWMP $\leq_{P}$ SP. is provided in Appendix A. From these, the computational equivalence of the SP and MWMP problems follows directly.

Let $C_{\mathrm{SP}}(|\mathcal{U}|,|\mathcal{V}|,|\mathcal{T}|)$ and $C_{\mathrm{MWMP}}(n)$ be the complexity of the SP problem and of the MWMP problem, respectively. The variable $n$ denotes the number of vertexes.

Corollary 1: The following upper bound to the complexity of the SP problem holds:

$$
\begin{aligned}
& C_{\mathrm{SP}}(|\mathcal{U}|,|\mathcal{V}|,|\mathcal{T}|) \in \\
& \quad O\left(\max \left\{(|\mathcal{U}|+|\mathcal{V}|)^{2}|\mathcal{T}|, C_{\text {MWMP }}(2(|\mathcal{U}|+|\mathcal{V}|))\right\}\right)
\end{aligned}
$$

Proof: The proof is a direct consequence of Theorem 1, which proves the polynomial-time reducibility of SP to MWMP. Hence, any instance of SP can be solved by mapping it onto an instance of MWMP as described above, i.e. construct a bipartite graph $G=(V, E)\left(\operatorname{cost} O(|\mathcal{U}|+|\mathcal{V}|)^{2} \mid \mathcal{T}\right)$, find the optimal solution of MWMP over $G\left(\operatorname{cost} C_{\text {MWMP }}(2(|\mathcal{U}|+\right.$ $|\mathcal{V}|))$ ) and convert it to the corresponding solution of SP (cost $O(|\mathcal{U}||\mathcal{T}|))$. The thesis follows immediately by combining the costs of the three steps.

The above bounds can be refined by defining $C_{\mathrm{SP}}(n)$ as the complexity of the SP problem versus the total size of the input: $n=|\mathcal{U}|+|\mathcal{V}|+|\mathcal{T}|$. Since it is known [21] that the complexity of MWMP is smaller than $O\left(n^{3}\right)$, the following bound can be easily derived: $C_{\mathrm{SP}}(n) \in O\left(n^{3}\right)$. This bound is tight in the sense that it cannot be reduced without making assumptions on the relative size of the input variables. If, for example, the number of time epochs is assumed to be a fixed parameter, then the following tighter bound holds: $C_{\mathrm{SP}}(n) \in O\left(C_{\mathrm{MWMP}}(n)\right)$.

Corollary 2: With $C_{\mathrm{SP}}(n)$ and $C_{\mathrm{MWMP}}(n)$ defined above, the following holds:

$$
C_{\mathrm{SP}}(n) \in \Omega\left(C_{\mathrm{MWMP}}(\sqrt{n})\right)
$$

where $\Omega$ is defined according to Knuth definition, i.e. $f \in$ $\Omega(g)$ iff $g \in O(f)$.

Proof: Let $m$ be the number of vertexes in the MWMP problem. A trivial bound to $C_{\mathrm{MWMP}}(m)$ is the number of edges, $m^{2} / 4$. By using the same definitions in Appendix A, the sizes of the sets $\mathcal{U}, \mathcal{V}, \mathcal{T}$ can be expressed as:

$$
\begin{aligned}
|\mathcal{U}| & =m \\
|\mathcal{V}| & =m / 2 \\
|\mathcal{T}| & =\frac{m(m+2)}{4}+3 .
\end{aligned}
$$

Then, by virtue of Theorem 2, the following equality holds:

$$
\begin{aligned}
C_{\mathrm{MWMP}}(m) & \in O\left(\max \left\{\frac{m^{2}}{4}, C_{\mathrm{SP}}\left(m+\frac{m}{2}+\frac{m(m+2)}{4}+3\right)\right\}\right) \\
& \in O\left(\max \left\{m^{2}, C_{\mathrm{SP}}\left(m^{2}\right)\right\}\right) .
\end{aligned}
$$

Since, the complexity of SP is at least linear, it holds $O\left(\max \left\{m^{2}, C_{\mathrm{SP}}\left(m^{2}\right)\right\}\right)=O\left(C_{\mathrm{SP}}\left(m^{2}\right)\right)$ and then, by the definition of $\Omega$, we obtain

$$
C_{\mathrm{SP}}\left(m^{2}\right) \in \Omega\left(C_{\mathrm{MWMP}}(m)\right)
$$

By performing the variable substitution $n=\sqrt{m}$, we obtain the thesis. 
The exact complexity of MWMP is not known. The best lower bound in the literature for the MWMP is $C_{\text {MWMP }}(n)=$ $\Omega\left(n^{2}\right)$. Applying this bound to Corollary 2 results in the obvious lower bound $C_{\mathrm{SP}}(n)=\Omega(n)$. However, if a larger lower bound for MWMP were found, then the lower bound for SP would be similarly increased.

\section{Discussion on Problem Assumptions}

It is worth noting that the assumption of uninterruptibility of the battery recharge process stated in Section III-A can be relaxed by replacing the triplet $(u, v, t)$ with a tuple $\left(u, v, T_{u}\right)$, where $T_{u} \subset \mathcal{T}:|T|=\left\lceil\frac{e_{u}}{r}\right\rceil$ is a set of $\left\lceil\frac{e_{u}}{r}\right\rceil$ time intervals (not necessarily contiguous) within the optimization time span. The objective funtion (4), must be replaced by

$$
\min _{\overline{\mathcal{Z}} \subseteq \mathcal{Z}} \sum_{\left(u, v, T_{u}\right) \in \overline{\mathcal{Z}}} \sum_{k \in T_{u}} c_{k}
$$

Equations (8), and (9) must be replaced as follows. If $n_{1} \in N_{1}^{\mathcal{U}}$ and $n_{2} \in N_{2}^{\mathcal{V}}$, then

$$
w\left(n_{1}, n_{2}\right)=\min _{T_{u}: t_{n}^{a} \leq k \leq|\mathcal{T}| \forall k \in T_{u}} \sum_{k \in T_{u}} c_{k}
$$

While if $n_{1} \in N_{1}^{\mathcal{U}}, n_{2} \in N_{2}^{\mathcal{U}}$ and $t_{n_{1}}^{a} \leq t_{n_{2}}^{d}-\left\lceil\frac{e_{n_{1}}}{r}\right\rceil$, then

$$
w\left(n_{1}, n_{2}\right)=\min _{T_{u}: t_{n_{1}}^{a} \leq k \leq t_{n_{2}}} \sum_{k \in T_{u}} c_{k}
$$

Algorithm 1 can be applied by replacing lines 5-7 with a cycle which retrieves $\left\lceil\frac{e_{u}}{r}\right\rceil$ time intervals within the range $\left[\tilde{t}, t_{n_{2}}^{d}\right]$ such that the overall recharge cost equals $w\left(n_{1}, n_{2}\right)$. Therefore, the optimal scheduling can still be computed. However, since the proof of polynomial reducibility of MWMP to SP provided in Appendix A cannot be straightforwardly extended to an interruptible recharge scenario, no conclusion on the computational equivalence of SP and MWMP can be drawn in such case.

Conversely, the assumption of constant recharge rate for all the vehicles of the fleet stated in Section III-A is necessary to allow for the computation of the weights $w\left(n_{1}, n_{2}\right)$ without prior assignment of the user requests to specific vehicles. Therefore, the relaxation of such assumption prevents the conversion of an SP instance into an MWMP instance. Note that recently commercialized EV rechargers [22], [23] support multiple rates. Therefore, in the future, the recharge rates of the vehicles are expected to be tunable, which could ensure rate homogeneity in the fleet.

\section{Heuristic AND BEnCHMARK Scheduling APPROACHES}

As discussed in the previous Sections, the optimal solution to the SP can be obtained by solving the corresponding MWMP and converting the optimal matching to the optimal schedule. However, such approach requires full knowledge of the users' arrival and departure times, which is an unrealistic assumption for the majority of real world applications. Therefore, in this Section we propose an heuristic online algorithm which schedules the recharge periods of the EVs by relying on historical data about the users' travel patterns $\overline{\text { Algorithm } 3 \text { History-based Online Scheduling Approach for }}$ SP

Input: $t$ : the current time

Input: $\operatorname{dep}(\tau)$ : the number of vehicles departure requests at time $\tau$. This is exact for $\tau=t$ and an estimate for $\tau>t$.

Input: $V$ : the set of vehicles that are not traveling or in charge at time $t$

Input: $\operatorname{epochs}(v), \forall v \in V$ : the number of epochs necessary to recharge vehicle $v$.

Output: $\operatorname{start}(t)$ : the set of vehicles departing at time $t$

Output: charge $(t)$ : the set of vehicles starting their charge at time $t$

1: Instantiate the set of departure requests $D=\left\{d_{1} \ldots d_{|V|}\right\}$ of size $|V|$.

2: Let deptime $(d), \forall d \in D$ the departure time of request $d$

3: $j \leftarrow 1$

4: for $\tau \leftarrow t$ to $|\mathcal{T}|$ do

5: $\quad$ for $i \leftarrow 1$ to $\operatorname{dep}(\tau)$ do

6: $\quad$ deptime $\left(d_{j}\right) \leftarrow \tau ; j \leftarrow j+1$

7: $\quad$ if $j=|V|$ break

8: $\quad$ end for

9: end for

10: loop

11: $\quad$ Build the graph $G(V, D)$

12: $\quad$ Calculate the edge weights $w(v, d)$ according to (15)

13: $\quad$ while $\exists v \in V: w(v, d)=\infty \forall d \in D$ do

14: $\quad$ Remove $v$ from $V$.

15: $\quad$ Drop the element in $D$ having the largest departure time. If such departure time equals $t$, the departure request cannot be fulfilled.

\section{6: end while}

17: Compute the matching $\bar{E}$ of minimum weight over graph $G(v, d)$

18: $\quad$ if $\bar{E}$ includes an edge $(v, d)$ with infinite weight then

19: $\quad$ Remove $v$ from $V$ and $d$ from $D$.

20: else

21: $\quad$ exit loop

22: end if

23: end loop

24: $\operatorname{start}(t) \leftarrow\{v \in V:(v, d) \in \bar{E} \wedge \operatorname{deptime}(d)=t\}$

25: charge $(t) \leftarrow$ vehicles in $V$ whose edges in $\bar{E}$ has a weight implying starting to charge at $t$ plus the vertexes removed from $V$ in lines 14 and 19

and updates the schedules at every epoch according to the actual departures/arrivals, with the aim of minimizing the overall recharge cost. We call such heuristic history-based online scheduler. Moreover, we introduce a benchmark online approach which simply schedules the recharge of each vehicle immediately upon arrival to the recharging station without any attempt to minimize the recharge cost. This approach is called immediate-charge online scheduler.

\section{A. History-based Online Scheduling Approach}

The history-based online algorithm (see Alg. 3) assumes the knowledge at the current time $t$ of the users' expected departure rate for each epoch $\tau \in \mathcal{T}$ with $\tau>t$ and the 
actual departures for $\tau \leq t$. In the Algorithm we denote both quantities by $\operatorname{dep}(\tau)$. Based on the above information, the algorithm builds a list $D$ of departures (lines 3-9). The algorithm also considers a set $V$ of vehicles, including the fully-charged ones and the ones that are not in charge. The charging process is considered uninterruptible, therefore the vehicles in charge and the traveling vehicles are not considered available for scheduling.

First, the graph $G(V, D)$ is built with edge weights according to the following equation:

$$
\begin{aligned}
& w(v, d)= \\
& \begin{cases}0 & \text { if epochs }(v)=0 \\
\infty & \text { if } \Delta(d, v)<0 \wedge \operatorname{epochs}(v)>0 \\
\min _{t \leq \tau \leq \Delta(d, v)} \sum_{k=\tau} c_{k} & \text { if } \Delta(d, v) \geq 0 \wedge \operatorname{epochs}(v)>0\end{cases}
\end{aligned}
$$

where epochs $(v)$ denotes the number of epochs needed to recharge vehicle $v$ and $\Delta(d, v)=\operatorname{deptime}(d)-\operatorname{epochs}(v)$.

If such graph includes any node $v \in V$ whose edges have all infinite weight, then $v$ is removed along with the farthest departure (lines 13-16). This is the case in which a vehicle cannot be charged in time for any departure in the list.

Then, the algorithm searches for a minimum weight matching $\bar{E}$ over the graph $G(V, D)$. In case the solution includes some edges with infinite weight, one of them is randomly selected and removed, the corresponding vertexes (a vehicle and a departure request) are respectively removed from the sets $V$ and $D$ (lines 10-23). In case the departure time of the removed vertex $d \in D$ equals $t$, then such departure request cannot be fulfilled and the user remains unserved. This procedure is repeated until a matching with finite weight is identified (line 21).

Finally, the algorithm outputs the vehicles departing at time $t$ and the vehicles starting to charge at time $t$.

The vehicles connected to users departing at time $t$ in the matching $\bar{E}$ are the vehicles departing at $t$. Note that the difference between departure requests, $\operatorname{dep}(t)$, and the actual departures, $|\operatorname{start}(t)|$, is equal to the number of unsatisfied requests.

The set of vehicles starting to charge at $t$ is built by considering the each vehicle $v \in V$. If the cost of charging starting immediately equal to the weight of the edge $(v, d) \in \bar{E}$, then the vehicle $v$ starts to charge immediately. In addition, all the vehicles removed from the graph at lines 14 and 19, also start to charge immediately.

The complexity of the history-based algorithm is $O(|\mathcal{T}|$. $\left.|\mathcal{V}| \cdot C_{\text {MWMP }}(|\mathcal{V}|)\right)$.

The history-based algorithm can be straightforwardly enhanced to a scenario in which the users' departure times are known in advance (whereas arrival times and the amount of discharged energy remain unknown) by building the list $D$ using the actual departures.

\section{B. Immediate-Charge Online Scheduling Approach}

As a benchmark, we define the immediate-charge on-line scheduling which does not assume any future knowledge about the traveling requests of the users in terms of departure/arrival times and amount of energy usage during the travel. We keep the assumption that all the vehicles are fully charged at the beginning of the scheduling horizon. Whenever a travel request by user $u$ comes, it is assigned to a randomly chosen vehicle among the ones parked at the recharging station and currently not under recharge (i.e. with full battery level). Then, as soon as $v$ returns at time $t_{u}^{a}$ after serving user $u$, it is immediately recharged during the next $\left\lceil e_{u} / r\right\rceil$ consecutive epochs. Therefore, the complexity of the algorithm is $O(|\mathcal{U}|)$, i.e. shows linear dependency on the number of users. Note that, since each recharge is scheduled immediately after the vehicle's return without introducing any waiting time, this algorithm always provides a feasible solution with no unsatisfied users, given that the considered instance of SP admits at least one feasible scheduling.

\section{Performance Assessment}

In this Section we quantify the savings of the optimal scheduling with day-ahead knowledge w.r.t. the online approaches presented in Section IV.

Numerical results are obtained assuming a scenario of a fleet ranging from 20 to 500 vehicles equipped with a 22 $\mathrm{kWh}$ battery pack [22] (recharge rate of $2.5 \mathrm{~kW}$ ), and two set of 50 and 500 users. The departure time, arrival time and amount of energy required for the travel have been computed based on the TripChaining dataset [24], which records departure/arrival time and covered distance for thousands of tours, i.e. sequences of trips that are linked together (chained) between two anchored destinations (e.g. home and work). For our purposes, we considered only the subset of tours in which the two anchors are identical (e.g. home-to-home or work-towork tours). The overall battery discharge at the end of the tour has been computed proportionally to the total covered distance. Statistics used by the history-based approach have been computed by averaging data of the whole subset. Each daily departure/arrival pattern includes 500 tours (one per user) randomly chosen within the subset. Note that the dataset does not provide the date in which the trips occurred, therefore possible weekly/seasonal variations in the travel patterns could not be captured by our simulations.

The trend of the hourly energy cost on the day-ahead energy market has been obtained from [25], which provides the realtime locational marginal pricing for several bus location of an American regional transmission organization. Typically, such prices exhibits hourly variations. For the sake of easiness, we have considered daily prices at a single bus location.

Results have been averaged over 365 days. Each 24-hours period from 06:00 to 05:59 is divided in 96 epochs each 15 minutes long. We assume that in the time span between 00:00 and 5:59 no departure takes place, which ensures that the vehicles can be fully recharged on time for the next day. Note that, though in our simulations the battery capacity and recharge rate are assumed constant over time, variations in such parameters due to aging or to environmental conditions (e.g. external temperature) can be easily incorporated by updating their value at the beginning of each scheduling period. 
Table I compares the performance of the optimal day-ahead scheduling, which assumes full knowledge of the departure and arrival times of the users, and of the history-based algorithm, which assumes only knowledge of historical travel data, to the immediate-charge algorithm, which does not rely on any information about the future travelling patterns of the users. The comparison is done in terms of average recharge cost gap (i.e. the percent increase of the overall energy cost due to the online approaches w.r.t. the optimal day-ahead scheduling) and in terms of percentage of unserved users (i.e. the fraction of users who do not find a fully charged vehicle at the moment of their expected departure from the charging station $)^{2}$. Results with 500 users show that the immediatecharge strategy increases the total recharge cost by on average 40-64\%, with peaks up to almost five times the optimal schedules. The history-based approach provides the same results of the optimal approach when the number of vehicles equals the number of users, but also for smaller fleet sizes its performance closely approaches the optimal one (on average the cost is increased by $1-13 \%$ w.r.t the optimal scheduling). Moreover, the average percentage of users not served by the history-based approach never exceeds $0.3 \%$ for the considered scenarios. Such percentage is considerably reduced in case knowledge of the users' departure times is available (at most $0.003 \%$ for the considered instances). Moreover, knowing the users' departure times allows for a slight reduction in the recharge cost with respect to the case in which only historical data are available. The savings allowed by both the optimal and historybased approaches decrease when the fleet size decreases, since a small fleet imposes that each EV must sequentially serve multiple users, which reduces the periods in which the EV is plugged at the recharging station and limits the recharge cost minimization, which must be performed over shorter time spans.

Analogous results are reported in Table II for a set of 50 users and a fleet size ranging from 20 to 50 vehicles. As one could expect, the relative improvement depends mainly from the ratio between the number of users and the fleet size. The Table confirms this intuition: the average gap and the percentage of unserved users for the immediate-charge scheduling algorithm and for the enhanced history-based scheduling are basically the same than in Table I given the same numberof-users/fleet-size ratio. The only difference is for the historybased scheduling. In fact, due to the smaller number of users, the actual users' arrival process exhibits a larger variability in comparison to its mean. The historical information is then a worse predictor of the actual arrival process and performance are worse than in the first scenario.

An example of the daily recharge schedule is plotted in Fig. 2 for the optimal, history-based, and immediate-charge approaches, from which it results that the optimal and historybased scheduling tend to schedule most of the recharges in the early afternoon and during the night, when the energy cost is significantly lower. However, the history-based approach tends

\footnotetext{
${ }^{2}$ We estimated computational times in the order of milliseconds for the online immediate charge scheduling, and in the order of minutes for the online history-based/ enhenced history-based and the day-ahead approaches on a 2.5 $\mathrm{GHz}$ Intel Core i5 processor
}

TABLE I

Performance COMPARISON OF THE History-BASED AND IMMEDIATE-CHARGE SCHEDULING APPROACHES VS. THE OPTIMAL SCHEDULING, ASSUMING 500 USERS

\begin{tabular}{c||c|c|c}
\hline \hline$|\mathcal{V}|$ & Aver. Cost [\$] & Aver. Gap [\%] & Unserved Users [\%] \\
\hline \hline \multicolumn{3}{c}{ Immediate-charge scheduling } \\
\hline 500 & $135.25 \pm 13.01$ & 64.2 & 0 \\
400 & $134.83 \pm 12.68$ & 60.3 & 0 \\
300 & $135.55 \pm 12.79$ & 52.6 & 0 \\
200 & $136.64 \pm 13.06$ & 40.4 & 0 \\
\hline \multicolumn{3}{c}{ History-based scheduling } \\
\hline 500 & $83.05 \pm 6.40$ & 0.8 & 0 \\
400 & $86.80 \pm 6.42$ & 4.0 & 0.007 \\
300 & $95.44 \pm 7.51$ & 9.0 & 0.10 \\
200 & $107.00 \pm 9.06$ & 13.0 & 0.29 \\
\hline \multicolumn{4}{c}{ Enhanced history-based scheduling } \\
\hline 500 & $82.54 \pm 6.39$ & 0 & 0 \\
400 & $85.76 \pm 6.35$ & 2.7 & $<5 \times 10^{-6}$ \\
300 & $93.73 \pm 6.99$ & 7.7 & $<5 \times 10^{-6}$ \\
200 & $106.80 \pm 8.41$ & 12.4 & 0.003 \\
\hline \hline
\end{tabular}

95\% confidence intervals are reported for average recharge costs.

TABLE II

Performance Comparison of the History-Based and IMMEDIATE-CHARGE SCHEDULING APPROACHES VS. THE OPTIMAL SCHEDULING ASSUMING 50 USERS

\begin{tabular}{c||c|c|c}
\hline \hline$|\mathcal{V}|$ & Aver. Cost [\$] & Aver. Gap [\%] & Unserved Users [\%] \\
\hline \hline \multicolumn{3}{c}{ Immediate-charge scheduling } \\
\hline 50 & $13.56 \pm 1.30$ & 64.4 & 0 \\
40 & $13.72 \pm 1.31$ & 60.4 & 0 \\
30 & $13.45 \pm 1.31$ & 52.3 & 0 \\
20 & $13.72 \pm 1.34$ & 39.6 & 0 \\
\hline \multicolumn{3}{c}{ History-based scheduling } \\
\hline 50 & $9.36 \pm 0.74$ & 12.8 & 0 \\
40 & $10.17 \pm 0.87$ & 18.5 & $5 \times 10^{-3}$ \\
30 & $10.73 \pm 1.02$ & 22.1 & 0.02 \\
20 & $11.98 \pm 1.08$ & 23.4 & 0.74 \\
\hline \multicolumn{4}{c}{ Enhanced history-based scheduling } \\
\hline 50 & $8.33 \pm 0.64$ & 0 & 0 \\
40 & $8.78 \pm 0.69$ & 2.6 & 0 \\
30 & $9.39 \pm 0.82$ & 7.5 & 0 \\
20 & $10.91 \pm 0.95$ & 12.8 & 0.03 \\
\hline \hline
\end{tabular}

$95 \%$ confidence intervals are reported for average recharge costs.

to anticipate the recharge of some vehicles, in order to keep some fully recharged EVs parked, which can be used when the number of users' departures exceeds the estimates based on the historical data. The effect of such strategy on EVs' availability is shown in Fig. 3, which shows the time evolution of the number of EVs parked at the recharging station (either fully charged, in charge or uncharged) and of those traveling.

In order to evaluate the effects of ageing, we also computed the overall distance covered by a vehicle during 365 days. Table III compares the average distance covered by the fleet and its standard deviation obtained with the Immediate-Charge online scheduling approach, first according to the version 


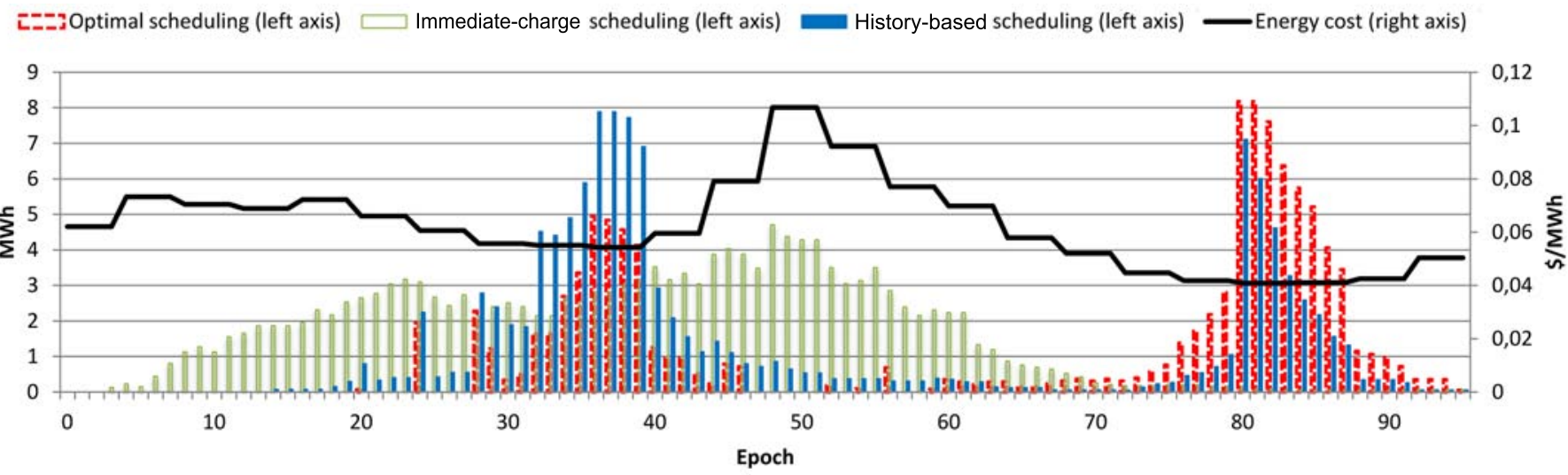

Fig. 2. Daily trend of overall scheduled recharge (optimal, history-based and immediate-charge approaches) vs. energy cost, assuming $|\mathcal{V}|=200,|\mathcal{U}|=500$.

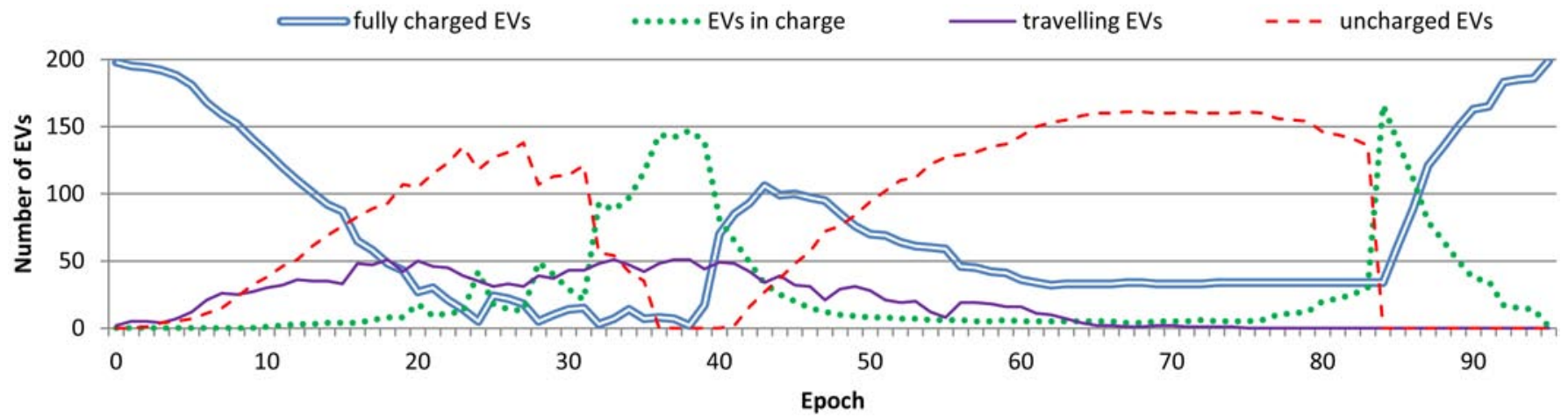

Fig. 3. Daily trend of the number of fully charged, in charge, uncharged and travelling EVs obtained by means of the history-based scheduling algorithm, assuming $|\mathcal{V}|=200,|\mathcal{U}|=500$.

TABLE III

Evaluation of Average Covered Distance

\begin{tabular}{c||c|cc}
\hline \hline & & Imm. Charge & $\begin{array}{c}\text { Enhanced Imm. Charge } \\
\text { Standard Dev. }\end{array}$ \\
\hline \hline V $\mid$ & Aver. Distance [km] & Standard Dev. & 25.7 \\
400 & 6499.9 & 493.7 & 26.9 \\
300 & 8159.4 & 500.7 & 28.0 \\
200 & 10834.8 & 635.8 & 27.8 \\
\hline \hline
\end{tabular}

Results assuming $|\mathcal{U}|=500$.

described in Section IV-B, then by applying an enhanced version which assigns to a user the vehicle with the lowest total covered distance chosen among the set of fully charged vehicles. Results show that introducing an ageing-aware policy allows for a reduction of one order of magnitude in the standard deviation of the covered distance (hundreds versus tens of $\mathrm{km}$ for the standard versus the enhanced scheduling). However, we believe that variations within the range of a few thousands of $\mathrm{km}$ per year in the distances covered by the fleet vehicles (which are achieved by our proposed scheduling mechanism) are sufficiently low to conclude that the ageing process is quite homogeneous.

\section{CONCLUSIONS}

This paper evaluates the computational complexity of an algorithm for optimal day-ahead scheduling of the battery recharge of a fleet of electric vehicles. We provide analytical upper and lower bounds to the problem complexity and compare the performance of the proposed algorithm to an online heuristic and a benchmark approach, showing that both the optimal and suboptimal methods lead to significant cost savings w.r.t. the naive benchmark. In case of our historybased heuristic approach, the price to be paid is a very low probability of service unavailability experienced by the users.

\section{APPENDIX A}

Proof of Polynomial Reducibility of MWMP to SP

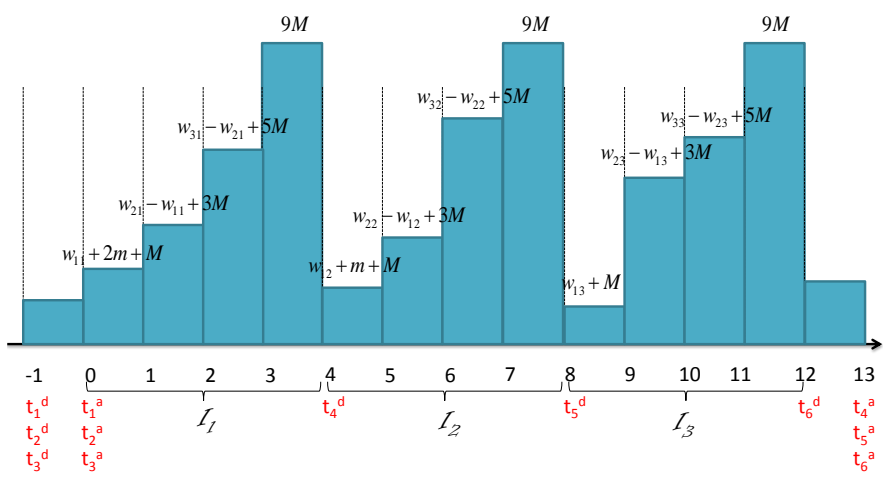

Fig. 4. Cost function obtained by means of Equation 17 from a bipartite complete balanced graph $G=(V, E)$ with $|V|=6$.

Let $G=\left(N_{1}, N_{2}\right)$ be a complete balanced bipartite graph as defined in Section II. In order to map a generic instance 
of MWMP with finite weights onto an instance of SP, consider $\left|N_{1}\right|$ vehicles $\left(\mathcal{V}=\left\{1, \ldots,\left|N_{1}\right|\right\}\right)$ and $2\left|N_{1}\right|$ users $\left(\mathcal{U}=\left\{1, \ldots, 2\left|N_{1}\right|\right\}\right)$. Let $\mathcal{T}=\left\{-1,0, \ldots,\left|N_{1}\right|^{2}+\left|N_{1}\right|+1\right\}$ be the set of time slots within the optimization horizon ${ }^{3}$. In the horizon we can distinguish the first and last time slots and $\left|N_{1}\right|$ intermediate intervals $\mathcal{I}_{h}\left(1 \leq h \leq\left|N_{1}\right|\right)$, each of length $\left|N_{1}\right|+1$ slots. Figure 4 shows such instance for $\left|N_{1}\right|=6$. A half of the users depart at the begin of timeslot -1 and return by the begin of time slot 0 (for $u \in \mathcal{U}: 1 \leq u \leq\left|N_{1}\right|$, $t_{u}^{d}=-1, t_{u}^{a}=0$ ), the other half of users departs later on (one user at the end of every intermediate interval) and returns at the end of the scheduling period (for $u \in \mathcal{U}:\left|N_{1}\right|+1 \leq u \leq$ $\left.2\left|N_{1}\right|, t_{u}^{d}=\left(u-\left|N_{1}\right|\right)\left(\left|N_{1}\right|+1\right), t_{u}^{a}=\left|N_{1}\right|^{2}+\left|N_{1}\right|+1\right)$.

By construction, it follows that each vehicle will be used by two and only two users. Since all the vehicles are back at the begin of timeslot 0 , this SP instance is equivalent to decide how to match the $\left|N_{1}\right|$ vehicles with the $\left|N_{1}\right|$ users departing later on. The rest of the proof will show that it is possible to determine energy costs so that the SP instance is able to capture all the variability of the $\left|N_{1}\right|^{2}$ weights of the MWMP instance.

In order to decouple the total energy recharge cost for the different vehicles, we can tune the required amount of energy to be recharged. In particular, the vehicle used by user $u \in$ $\mathcal{U}: 1 \leq u \leq\left|N_{1}\right|$ requires $e_{u}=u r$. For the second half of users we can consider arbitrary energy requests (e.g. $e_{u}=0$ for $u \in \mathcal{U}:\left|N_{1}\right|+1 \leq u \leq 2\left|N_{1}\right|$ ).

Let us denote by $m$ and $M$ two positive numbers such that $m>\max _{\left(n_{1}, n_{2}\right)} w\left(n_{1}, n_{2}\right), M>m\left|N_{1}\right|$. We first observe that if all the weights of the links outgoing from a given node $n_{1}$ in $N_{1}$ (or $n_{2}$ in $N_{2}$ ) are increased by the same amount $x_{n_{1}}$ (or $x_{n_{2}}$ ), the MWMP instance is transformed in an equivalent one with the same minimum weight matching (whose value will be increased by $x_{n_{1}}$ or $x_{n_{2}}$ ). We can apply this transformation for all the nodes, thus obtaining a sequence of equivalent MWMP instances. This reasoning leads us to conclude that if we transform the weight of each link as follows:

$$
w^{\prime}\left(n_{1}, n_{2}\right)=w\left(n_{1}, n_{2}\right)+M n_{1}^{2}+m\left(\left|N_{1}\right|-n_{2}\right),
$$

we obtain an equivalent MWMP, because each weight has been increased by two quantities that depend only on the two terminating nodes.

We define the energy cost $c_{\tau}$ as follows:

$$
c_{\tau}=\left\{\begin{array}{l}
w\left(1,\left\lceil\frac{\tau+1}{\left|N_{1}\right|+1}\right\rceil\right) \text { if } \tau=0 \vee \tau \bmod \left(\left|N_{1}\right|+1\right)=0 \\
M\left|N_{1}\right|^{2} \quad \text { if } \tau \bmod \left(\left|N_{1}\right|+1\right)=\left|N_{1}\right| \\
w^{\prime}\left(\tau \bmod \left(\left|N_{1}\right|+1\right)+1,\left\lceil\frac{\tau+1}{\left|N_{1}\right|+1}\right\rceil\right)+ \\
\quad-w^{\prime}\left(\tau \bmod \left(\left|N_{1}\right|+1\right),\left\lceil\frac{\tau+1}{\left|N_{1}\right|+1}\right\rceil\right) \text { otherwise }
\end{array}\right.
$$

We observe that the computation of $c_{t a u}$ starting from the weights $w\left(n_{1}, n_{2}\right)$ requires $O\left(\left|N_{1}\right|^{2}\right)$ operations.

It can be checked that this definition of the costs leads to the following consequences (the reader may find useful to refer to Figure 4). First, because of the presence of the

\footnotetext{
${ }^{3}$ For ease of notation we set the first epoch to -1 , but the mapping from the MWMP instance to the corresponding SP instance could be performed considering any arbitrary time horizon of $\left|N_{1}\right|^{2}+\left|N_{1}\right|+1+3$ epochs
}

large constant $M$, energy costs are always increasing within each intermediate interval. Moreover, the presence of slots with cost $M\left|N_{1}\right|^{2}$ at the end of each intermediate interval makes inconvenient for the recharge of a vehicle to span two consecutive intervals. As a result, every vehicle will be charged by starting only at the begin of an interval. In particular, if the vehicle driven by user $n_{1} \in\left\{1, \ldots\left|N_{1}\right|\right\}$ is charged during the interval $\mathcal{I}_{h}$, the aggregate recharge cost is: $\sum_{k=(h-1)\left(\left|N_{1}\right|+1\right)}^{(h-1)\left(\mid N_{1}+1\right)+n} c_{k}=w_{e\left(n_{1}, h\right)}^{\prime}$, i.e. equal to the weight of link $e\left(n_{1}, h\right)$ in the equivalent MWMP we are considering. Second, the presence of the constant $m$ in the weight function guarantees that the vehicle to be driven by user $u \in\left\{\left|N_{1}\right|+1, \ldots 2\left|N_{1}\right|\right\}$ will not be charged before the interval $\mathcal{I}_{u-\left|N_{1}\right|}$. As a consequence, only one vehicle will be charged during each interval.

In conclusion this SP instance is equivalent to match each of the $\left|N_{1}\right|$ vehicles to a different recharging interval.

Consider a matching $\bar{E}$ over graph $G$ such that $\sum_{\left(n_{1}, n_{2}\right) \in \bar{E}} w\left(n_{1}, n_{2}\right)=\Xi$. Then, by construction there always exists a feasible schedule $\overline{\mathcal{Z}}$ defined as:

$$
\overline{\mathcal{Z}}=\bigcup_{\left(n_{1}, n_{2}\right) \in \bar{E}}\left\{\left(n_{1}, n_{1},\left(\left|N_{1}\right|+1\right)\left(n_{2}-1\right)\right),\left(\left(n_{2}+\left|N_{1}\right|, n_{1},|\mathcal{T}|\right)\right\}\right.
$$

. Note that, since users $\left|N_{1}\right|+1, \ldots, 2\left|N_{1}\right|$ do not consume any energy during their travel, the associated recharging periods last 0 epochs and are scheduled at $\tau=|\mathcal{T}|$.

From Eqs. (16), (17) it follows that:

$$
\begin{array}{r}
\sum_{(u, v, t) \in \overline{\mathcal{Z}}} \sum_{k=t}^{t+\left\lceil e_{u} / r\right\rceil-1} c_{k}=\sum_{\left(n_{1}, n_{2}\right) \in \bar{E}} w\left(n_{1}, n_{2}\right)= \\
\sum_{\substack{\left.\mid n_{1}, n_{2}\right) \in \bar{E} \\
\Xi+m}} w\left(n_{1}, n_{2}\right)+\sum_{i=1}^{\left|N_{1}\right|}\left((i-1) m+i^{2} M\right)= \\
\hline
\end{array}
$$

i.e. the cost of the schedule $\overline{\mathcal{Z}}$ is obtained by adding to the cost of the matching $\bar{E}$ a positive quantity which depends only on the cardinality of $N_{1}$.

Consider now an arbitrary scheduling $\overline{\mathcal{Z}}$ defined over an instance of SP constructed as discussed at the beginning of this Section. It is worth noting that, for a given association between users and vehicles, the schedule of lowest cost imposes that each vehicle is recharged in a different time interval $\mathcal{I}_{h}$, starting from the first time slot of the interval. This can be shown by considering any pair of triplets $(u, v, t),\left(u^{\prime}, v^{\prime}, t^{\prime}\right): u \leq\left|N_{1}\right|, u^{\prime}>\left|N_{1}\right|$. By construction the following equality holds:

$$
\begin{aligned}
w^{\prime}\left(n_{1}, n_{2}\right) & =\sum_{k=\left(n_{2}-1\right)\left(\left|N_{1}\right|+1\right)}^{k=\left(n_{2}-1\right)\left(\left|N_{1}\right|+1\right)+n_{1}-1} c_{k} \\
& =\min _{t \in \mathcal{T}: 0 \leq t<\left(\left|N_{1}\right|+1\right) n_{2}} \sum_{k=t}^{t+n_{1}-1} c_{k}
\end{aligned}
$$


where $n_{1}=u, n_{2}=u^{\prime}-\left|N_{1}\right|$. Let $\bar{n}_{2}=\left\lceil\frac{t+1}{\left|N_{1}\right|+1}\right\rceil$. We start with $n_{1}=1$ and distinguish the following three cases:

1) if $t \bmod \left(\left|N_{1}\right|+1\right)=0$, we get $\sum_{k=t}^{t+\left\lceil\frac{e_{u}}{r}\right\rceil-1} c_{k}=$ $w_{e\left(1, \bar{n}_{2}\right)}^{\prime}$. By applying Formula 16 the inequality

$$
w^{\prime}\left(1, \bar{n}_{2}\right) \geq w^{\prime}\left(1, n_{2}\right)
$$

results in:

$$
w\left(1, \bar{n}_{2}\right)+m\left(n_{2}-\bar{n}_{2}\right) \geq w\left(1, n_{2}\right)
$$

which always holds, since $m>\max _{\left(n_{1}, n_{2}\right)} w\left(n_{1}, n_{2}\right)$ by construction and $t<n_{2}\left(\left|N_{1}\right|+1\right)$ implies that $\bar{n}_{2} \leq$ $n_{2}$.

2) if $t \bmod \left(\left|N_{1}\right|+1\right)=\left|N_{1}\right|$, we get $\sum_{k=t}^{t+\left\lceil\frac{e u}{r}\right\rceil-1} c_{k}=$ $M\left|N_{1}\right|^{2}$. The inequality resulting from Eq. 19 by substitution is:

$$
M\left(\left|N_{1}\right|^{2}-1\right) \geq w\left(1, n_{2}\right)+m\left(\left|N_{1}\right|-n_{2}\right)
$$

which always holds by construction, since $m>$ $\max _{\left(n_{1}, n_{2}\right)} w\left(n_{1}, n_{2}\right), M>m\left|N_{1}\right|$.

3) if $t \bmod \left(\left|N_{1}\right|+1\right) \neq\left|N_{1}\right| \wedge t \bmod \left(\left|N_{1}\right|+1\right) \neq 0$, we obtain $\sum_{k=t}^{t+\left\lceil\frac{e_{u}}{r}\right\rceil-1} c_{k}=w^{\prime}\left(\bar{n}_{1}+1, \bar{n}_{2}\right)-w^{\prime}\left(\bar{n}_{1}, \bar{n}_{2}\right)$. By substitution, from Eq. 19 it results:

$$
\begin{aligned}
& w\left(\bar{n}_{1}+1, \bar{n}_{2}\right)+M\left(1+2 \bar{n}_{1}\right) \geq \\
& w\left(\bar{n}_{1}, \bar{n}_{2}\right)+w\left(n_{1}, n_{2}\right)+m\left(\left|N_{1}\right|-n_{2}\right)
\end{aligned}
$$

which always holds by construction, since $m>$ $\max _{\left(n_{1}, n_{2}\right)} w\left(n_{1}, n_{2}\right), M>m\left|N_{1}\right|$.

The generalization for $n_{1}>1$ is straightforward.

It follows that, considering an arbitrary association between vehicles and users as defined by the scheduling $\overline{\mathcal{Z}}$ of cost $\Psi=$ $\sum_{(u, v, t) \in \overline{\mathcal{Z}}} \sum_{k=t}^{t+\left\lceil e_{u} / r\right\rceil-1} c_{k}$, a scheduling $\overline{\mathcal{Z}}^{\prime}$ which allocates $\left|N_{1}\right|$ recharge periods of $1, \ldots,\left|N_{1}\right|$ slots respectively, each of them starting at the beginning of a different interval $\mathcal{I}_{h}$, results in a cost $\Psi^{\prime} \leq \Psi$. Since our objective is the minimization of the recharge cost, in the rest of the proof we will consider schedules which satisfy the above stated condition. Under such assumption, the matching $\bar{E}$ corresponding to $\overline{\mathcal{Z}}^{\prime}$ can be computed as follows:

$$
\bar{E}=\left\{\left(u, u^{\prime}-\left|N_{1}\right|\right) \quad \forall(u, v, t),\left(u^{\prime}, v^{\prime}, t^{\prime}\right) \in \overline{\mathcal{Z}}^{\prime}: v=v^{\prime}\right\}
$$

and, by construction, its cost is:

$$
\begin{aligned}
& \sum_{\left(n_{1}, n_{2}\right) \in \bar{E}} w\left(n_{1}, n_{2}\right)=\Psi^{\prime}- m \frac{\left|N_{1}\right|}{2}\left(\left|N_{1}\right|-1\right)- \\
& M\left(\frac{\left|N_{1}\right|^{3}}{3}+\frac{\left|N_{1}\right|^{2}}{2}+\frac{\left|N_{1}\right|}{6}\right) .
\end{aligned}
$$

Let now $\bar{E}_{\text {opt }}$ be the matching of minimum cost $\Xi$ of a generic instance of MWMP. Compute the scheduling $\overline{\mathcal{Z}}_{\text {opt }}$ of the equivalent SP instance by means of Eq. 18, which has cost $\Xi+m \frac{\left|N_{1}\right|}{2}\left(\left|N_{1}\right|-1\right)+M\left(\frac{\left|N_{1}\right|^{3}}{3}+\frac{\left|N_{1}\right|^{2}}{2}+\frac{\left|N_{1}\right|}{6}\right)$. If $\overline{\mathcal{Z}}_{\text {opt }}$ is not optimal, then there exists a solution $\overline{\mathcal{Z}}_{\text {opt }}^{*}$ of $\operatorname{cost} \Psi<\Xi+m \frac{\left|N_{1}\right|}{2}\left(\left|N_{1}\right|-1\right)+M\left(\frac{\left|N_{1}\right|^{3}}{3}+\frac{\left|N_{1}\right|^{2}}{2}+\frac{\left|N_{1}\right|}{6}\right)$, which is the optimal one $\overline{\mathcal{Z}}_{\text {opt }}^{*}$ and thus respects the condition that each recharge period starts at the beginning of a different time interval, which allows for the application of Eq. 20. Map $\overline{\mathcal{Z}}_{\text {opt }}^{*}$ onto the corresponding matching $\bar{E}_{\text {opt }}^{*}$ by means of Eq.20. By construction, such matching has cost $\Psi-m \frac{\left|N_{1}\right|}{2}\left(\left|N_{1}\right|-1\right)-M\left(\frac{\left|N_{1}\right|^{3}}{3}+\frac{\left|N_{1}\right|^{2}}{2}+\frac{\left|N_{1}\right|}{6}\right)<\Xi$. It follows that $\bar{E}_{\text {opt }}$ is not the matching of minimum weight, which contradicts the hypothesis.

Similarly, let $\overline{\mathcal{Z}}_{\text {opt }}$ be the optimal scheduling of an instance of SP constructed as discussed at the beginning of this Section, with cost $\Gamma$. Since such scheduling is optimal, it must allocate each recharge period at the beginning of a different time interval, thus Eq. 20 can be applied. Convert $\overline{\mathcal{Z}}_{\text {opt }}$ into $\bar{E}_{\text {opt }}$ of the equivalent MWMP instance by means of Eq. 20, thus obtaining a matching of cost $\Gamma-m \frac{\left|N_{1}\right|}{2}\left(\left|N_{1}\right|-1\right)-M\left(\frac{\left|N_{1}\right|^{3}}{3}+\frac{\left|N_{1}\right|^{2}}{2}+\frac{\left|N_{1}\right|}{6}\right)$. If $\bar{E}_{\text {opt }}$ is not the matching of minimum weight, then there must be another matching $\bar{E}_{\text {opt }}^{*}$ of weight $\Delta<\Gamma-m \frac{\left|N_{1}\right|}{2}\left(\left|N_{1}\right|-1\right)-$ $M\left(\frac{\left|N_{1}\right|^{3}}{3}+\frac{\left|N_{1}\right|^{2}}{2}+\frac{\left|N_{1}\right|}{6}\right)$. Map $\bar{E}_{\text {opt }}^{*}$ onto the corresponding scheduling $\overline{\mathcal{Z}}_{\text {opt }}^{*}$ by means of Eq. 18. It follows that the cost of $\overline{\mathcal{Z}}_{\text {opt }}^{*}$ is $\Delta+m \frac{\left|N_{1}\right|}{2}\left(\left|N_{1}\right|-1\right)+M\left(\frac{\left|N_{1}\right|^{3}}{3}+\frac{\left|N_{1}\right|^{2}}{2}+\frac{\left|N_{1}\right|}{6}\right)<$ $\Gamma$, therefore $\overline{\mathcal{Z}}_{\text {opt }}$ is not optimal, which contradicts the hypothesis.

It follows that $\overline{\mathcal{Z}}_{\text {opt }}$ is the optimal scheduling iff $\bar{E}_{\text {opt }}$ is the matching of minimum weight.

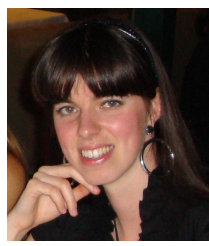

Cristina Rottondi received both Master's and Ph.D. Degrees cum laude in Telecommuni- cations Engineering from Politecnico di Milano in 2010 and 2014 respectively. She is currently postdoctoral researcher in the Department of Electronics, Information, and Bioengineering (DEIB) of Politecnico di Milano. Her research interests include communication security in the metering infrastructure of smart grids, energy management in smart buildings, and optical networks planning.

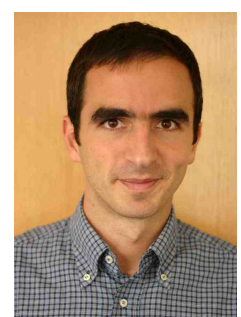

Giovanni Neglia received the Master's degree in electronic engineering and the Ph.D. degree in telecommunications from the University of Palermo, Palermo, Italy, in 2001 and 2005, respectively. In 2005, he was a Research Scholar with the University of Massachusetts, Amherst, visiting the Computer Networks Research Group. Before joining the National Research Institute of Informatics and Control (INRIA), Sophia-Antipolis, France, as a permanent member in September 2008, he became a Postdoctoral Researcher with the University of Palermo in June 2005 and an External Scientific Advisor with the Maestro team in September 2006. He is currently a Researcher with the Maestro team, INRIA. To this end, he applies different mathematical tools (Markov processes, control theory, fluid models, game theory, and gradient-based and metaheuristic optimization). His research interests include modeling and performance evaluation of computer networks and proposals of new mechanisms to improve their performance. 


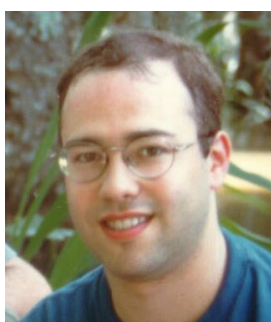

Giacomo Verticale is Researcher at Politecnico di Milano, Italy. He is co-head of the Broadband Optical Networks, Security and Advanced Internet (BONSAI) Laboratory in the Department of Electronics, Information, and Bioengineering (DEIB). Before joining Politecnico di Milano, he was with the CEFRIEL research center. He graduated in 2003 at Politecnico di Milano defending a thesis on the performance of packet transmission in $3 \mathrm{G}$ mobile networks. His research interests are in network security and in performance evaluation of network

protocols

\section{REFERENCES}

[1] J. Lopes, F. Soares, and P. Almeida, "Integration of electric vehicles in the electric power system," Proceedings of the IEEE, vol. 99, no. 1, pp. 168-183, 2011.

[2] T. Markel, M. Kuss, and P. Denholm, "Communication and control of electric drive vehicles supporting renewables," in Vehicle Power and Propulsion Conference, IEEE, 2009, pp. 27-34.

[3] A. Brooks, "Integration of electric drive vehicles with the power grida new application for vehicle batteries," in Battery Conference on Applications and Advances, The Seventeenth Annual, 2002, pp. 239-

[4] W. Kempton, F. Marra, P. Andersen, and R. Garcia-Valle, "Business models and control and management architectures for ev electrical grid integration," in Electric Vehicle Integration into Modern Power Networks, ser. Power Electronics and Power Systems, R. Garcia-Valle and J. A. Peas Lopes, Eds. Springer New York, 2013, pp. 87-105.

[5] R. J. Bessa and M. A. Matos, "Economic and technical management of an aggregation agent for electric vehicles: a literature survey," European Transactions on Electrical Power, vol. 22, no. 3, pp. 334-350, 2012.

[6] E. Pagliara, G. Parlangeli, T. Donateo, and F. Adamo, "Real time implementation of an optimal power management strategy for a plug-in hybrid electric vehicle," in Decision and Control, 52nd IEEE Conference on, Dec 2013.

[7] R. Patil, J. Kelly, Z. Filipi, and H. Fathy, "A framework for the integrated optimization of charging and power management in plug-in hybrid electric vehicles," Vehicular Technology, IEEE Transactions on, vol. 62, no. 6, pp. 2402-2412, July 2013.

[8] Y. Han, Y. Chen, F. Han, and K. Liu, "An optimal dynamic pricing and schedule approach in v2g," in Signal Information Processing Association Annual Summit and Conference (APSIPA ASC), 2012 Asia-Pacific, 2012, pp. 1-8.

[9] Y. He, B. Venkatesh, and L. Guan, "Optimal scheduling for charging and discharging of electric vehicles," Smart Grid, IEEE Transactions on, vol. 3, no. 3, pp. 1095-1105, 2012.

[10] G. Li and X.-P. Zhang, "Modeling of plug-in hybrid electric vehicle charging demand in probabilistic power flow calculations," Smart Grid, IEEE Transactions on, vol. 3, no. 1, pp. 492-499, 2012.

[11] C. Rottondi, S. Fontana, and G. Verticale, "Enabling privacy in vehicleto-grid interactions for battery recharging," Energies, vol. 7, no. 5, pp. 2780-2798, 2014. [Online]. Available: http://www.mdpi.com/1996$1073 / 7 / 5 / 2780$

[12] A. Di Giorgio, F. Liberati, and A. Pietrabissa, "On-board stochastic control of electric vehicle recharging," in Decision and Control, 52nd IEEE Conference on, Dec 2013.

[13] R. Liu, L. Dow, and E. Liu, "A survey of pev impacts on electric utilities," in Innovative Smart Grid Technologies (ISGT), 2011 IEEE PES, 2011, pp. 1-8.

[14] M. Alizadeh, A. Scaglione, J. Davies, and K. Kurani, "A scalable stochastic model for the electricity demand of electric and plug-in hybrid vehicles," Smart Grid, IEEE Transactions on, vol. PP, no. 99, pp. 1-13, 2013.

[15] S. Zou, Z. Ma, and X. Liu, "Distributed efficient charging coordinations for electric vehicles under progressive second price auction mechanism," in Decision and Control, 52nd IEEE Conference on, Dec 2013.

[16] J. Rivera, P. Wolfrum, S. Hirche, C. Goebel, and H.-A. Jacobsen, "Alternating direction method of multipliers for decentralized electric vehicle charging control," in Decision and Control, 52nd IEEE Conference on, Dec 2013

[17] C. Joe-Wong, S. Sen, S. Ha, and M. Chiang, "Optimized day-ahead pricing for smart grids with device-specific scheduling flexibility," Selected Areas in Communications, IEEE Journal on, vol. 30, no. 6, pp. 1075-1085, 2012.
[18] C. Jin, J. Tang, and P. Ghosh, "Optimizing electric vehicle charging: A customer's perspective," Vehicular Technology, IEEE Transactions on, vol. 62, no. 7, pp. 2919-2927, Sept 2013.

[19] T. Zhang, W. Chen, Z. Han, and Z. Cao, "Charging scheduling of electric vehicles with local renewable energy under uncertain electric vehicle arrival and grid power price," Vehicular Technology, IEEE Transactions on, vol. PP, no. 99, pp. 1-1, 2013.

[20] H. W. Kuhn, "The hungarian method for the assignment problem," Naval Research Logistics Quarterly, vol. 2, no. 1-2, pp. 83-97, 1955.

[21] R. Duan and S. Pettie, "Approximating maximum weight matching in near-linear time," in Foundations of Computer Science (FOCS), 2010 51st Annual IEEE Symposium on, Oct 2010, pp. 673-682.

[22] "Green car congress: Energy, technology, issues and policies for sustainable mobility," 2012. [Online]. Available: http://www.greencarcongress.com/2012/03/zoe-20120309.html

[23] S. Loudot, B. Briane, O. Ploix, and A. Villeneuve, "Fast charging device for an electric vehicle," Nov. 15 2012, uS Patent App. 12/919,396. [Online]. Available: http://www.google.com/patents/US20120286740

[24] U.S. Department of Transportation, Federal Highway Administration, "National household travel survey," 2009. [Online]. Available: http://nhts.ornl.gov

[25] "Daily real-time locational marginal pricing," 2013. [Online]. Available: http://www.pjm.com/markets-and-operations/energy/real-time/lmp.aspx 